\title{
Explorando Robótica com Pensamento Computacional no Ensino Médio: Um estudo sobre seus efeitos na educação
}

\author{
Isabelle M. L. Souza ${ }^{1}$, Rivanilson S. Rodrigues ${ }^{2}$, Wilkerson L. Andrade ${ }^{2}$ \\ ${ }^{1}$ Serviço Social da Indústria (SESI), Campina Grande, Brasil \\ ${ }^{2}$ Universidade Federal de Campina Grande (UFCG), Campina Grande, Brasil \\ isabellelimalfiepb.org.br, rivanilson.sbegmail.com, \\ wilkersonecomputacao.ufcg.edu.br
}

\begin{abstract}
Robotics workshops for basic education are often proposed for the improvement of teaching practices in different disciplines. However, the teaching of these workshops is too difficult given the deficiency of teachers and students education in Computational Thinking (CT) skills. Then, in order to improve the teaching and learning process in these workshops we propose a robotics course with emphasis on CT for High School in Paraíba SESI Schools. This work shows the statistical analysis of the effects of robotics workshop in the performance of 729 students approved in the 1st year of SESI High School. The results show that student performance was significantly improved after introducing CT on curriculum of robotics workshop.
\end{abstract}

\begin{abstract}
Resumo. Oficinas de robótica para educação básica frequentemente são propostas com vista ao aprimoramento de práticas de ensino em diferentes disciplinas. Contudo, o ensino dessas oficinas é demasiadamente dificultado diante da deficiência na formação docente e discente em competências do Pensamento Computacional (PC). Nesse sentido, com o intuito de melhorar o processo de ensino-aprendizagem nessas oficinas foi realizado um curso de robótica com ênfase em PC para o Ensino Médio nas Escolas SESI Paraíba. Avaliamos estatisticamente os efeitos do referido curso no desempenho de 729 estudantes aprovados no $1^{\circ}$ ano do Ensino Médio. Os resultados apontam que o desempenho dos alunos foi significativamente aprimorado após o curso.
\end{abstract}

\section{Introdução}

O Sistema de Ensino Brasileiro (SEB) definiu, através de diretrizes, que a formação do aluno necessita ser baseada na contextualização, criticidade, autonomia e aliança entre teoria e prática. Para tanto, é imprescindível definir práticas pedagógicas para concretizar essas indicações no Ensino Básico. Oliveira (1999) apresenta a educação como uma linha interdisciplinar e aplicada, alimentada por conceitos teóricos, construídos na prática e derivados da união de várias disciplinas do currículo.

Para Rodrigues et al. (2015), a inserção do Pensamento Computacional (PC) no Ensino Básico pode aprimorar a performance dos alunos nas áreas do conhecimento que tenham relações com situações de resolução de problemas. Para Wing (2006), trabalhar com PC é abordar noções de estruturas da computação, algoritmos, problemas, lógica, entre outras habilidades. Aguiar et al. (2015) destaca que as habilidades de raciocínio lógico são impulsionadas ao serem ofertadas junto ao ensino com robótica. 
V Congresso Brasileiro de Informática na Educação (CBIE 2016)

Anais do XXVII Simpósio Brasileiro de Informática na Educação (SBIE 2016)

Nesse contexto, o Serviço Social da Indústria, Departamento Regional da Paraíba (SESI-PB) implantou o Programa Educação Básica articulada com Educação Profissional (EBEP) composto por um currículo diferenciado que alia as disciplinas da base comum às disciplinas transversais. Dentre as disciplinas transversais, destaca-se a Oficina Tecnológica de Robótica ofertada em 2014, aos alunos da $1^{a}$ Série com foco no ensino de física, através do Modelo LEGOß de Educação Tecnológica. Nesse formato, os alunos apresentaram dificuldades na etapa de programação dos robôs e a razão disso pode ter relação com a ausência do PC na metodologia da LEGO utilizada. Com isso, em 2015 foi oferecida aos alunos da $1^{\text {a }}$ Série uma Oficina pautada nos conceitos de Pensamento Computacional para o ensino de Robótica.

Nesse aspecto, a presente pesquisa tem por objetivo avaliar os efeitos da introdução de PC na disciplina de robótica e nas disciplinas da base comum do Ensino Médio (EM) do SESI-PB. Buscamos responder as seguintes perguntas: (RQ1) A introdução do Pensamento Computacional favorece o processo de ensino-aprendizagem na oficina de Robótica? (RQ2) O ensino de Robótica com ênfase em Pensamento Computacional pode aprimorar o desempenho de estudantes nas disciplinas básicas do Ensino Médio?

Realizamos um estudo baseado no método de pesquisa ação, dividido em duas fases: (1) Desenvolvimento e aplicação de um curso de introdução ao PC aplicado à robótica para professores e estudantes do Ensino Médio da rede SESI, (2) Análise do efeito do curso no desempenho de estudantes após a modificação da disciplina. Usamos uma amostra de 729 estudantes aprovados na $1^{\text {a }}$ Série do Ensino Médio do SESI-PB nos anos de 2014 e 2015. Avaliamos o desempenho dos alunos na disciplina de robótica e nas disciplinas da base comum por área do conhecimento com e sem o ensino do PC.

Os resultados indicaram que $89 \%$ dos alunos que realizaram a oficina com PC obtiveram melhor desempenho comparado com os que realizaram a oficina sem PC, demonstrando assim, que a inserção de PC melhora o desempenho de estudantes na Oficina. Além disso, a porcentagem de alunos que tiveram contato com o PC e obtiveram melhor desempenho comparado aos que não tiveram, por áreas do conhecimento foram: 63\% Matemática e suas Tecnologias, 67\% Linguagens e Códigos e suas Tecnologias, 88\% Ciências Humanas e suas Tecnologias, $70 \%$ Ciências da Natureza e suas Tecnologias; confirmando que, após a introdução de PC na Oficina de Robótica, o desempenho dos estudantes nas disciplinas básicas melhora significativamente.

Este trabalho segue organizado nas seguintes seções: Na Seção 2, apresentamos uma contextualização abordando as diretrizes da Educação Brasileira, o Pensamento Computacional, o Ensino Médio do SESI e a Metodologia LEGO; na Seção 3, descrevemos o método utilizado no incremento da pesquisa; na Seção 4, delineamos os resultados, discussão e ameaças à validade da pesquisa e por fim, na Seção 5, exibimos as principais conclusões e trabalhos futuros.

\section{Contextualização do Trabalho}

Nessa seção apresentamos aspectos importantes para compreensão do contexto e problema de pesquisa. 
V Congresso Brasileiro de Informática na Educação (CBIE 2016)

Anais do XXVII Simpósio Brasileiro de Informática na Educação (SBIE 2016)

\subsection{A Educação Básica Brasileira}

O SEB é responsável por organizar a Educação Básica (EB) no Brasil, dividindo-a em 3 etapas: Educação Infantil, Ensino Fundamental e Ensino Médio. Considerando os direcionamentos do Ministério da Educação (MEC), a principal questão a ser avaliada é o objetivo do EM de potencializar o senso crítico e autonomia intelectual do aluno. Com essas finalidades e diretrizes o SEB definiu o perfil do aluno ao término dessa etapa: autônomo, conhecedor de princípios científicos e tecnológicos, com domínio da linguagem e condições de angariar novos estágios do seu desenvolvimento intelectual.

Para avaliar a qualidade do EM, o MEC criou em 1998 o Exame Nacional do Ensino Médio (ENEM), composto por 180 questões de múltipla escolha, com provas divididas em 4 áreas de conhecimentos: Ciências Humanas e suas Tecnologias (História, Geografia, Filosofia e Sociologia), Ciências da Natureza e suas Tecnologias (Química, Física e Biologia), Linguagens, Códigos e suas Tecnologias (Língua Portuguesa, Literatura, Língua Estrangeira, Artes, Educação Física e Tecnologias da Informação e Comunicação) e Matemática e suas Tecnologias (INEP, 2016); além de uma prova de produção textual referente a um tema social, científico, cultural ou político.

\subsection{Pensamento Computacional no Contexto da Educação Básica Brasileira}

Diante das finalidades e diretrizes do SEB são necessárias medidas para que as escolas do país ofertem ações educativas com resultados positivos. Paiva (2015) destaca que a conexão entre interdisciplinaridade e contextualização é necessária para a oferta de um currículo como sinalizado nos Parâmetros Curriculares Nacionais nas Diretrizes Curriculares Nacionais da Educação Básica. Matos (2013) defende propostas interdisciplinares, que favoreça explorar a relação entre os múltiplos componentes curriculares, com ações educativas que envolva o aluno em questões complexas, de diversas origens, permitindo-o desenvolver autonomia, senso crítico, capacidade e habilidade de solucionar problemas através de raciocínio lógico aliado à linguagem.

Uma resposta à necessidade em destaque foi oferecida pelo ACM Model Curriculum for K-12 Computer Science (CSTA, 2016) ao defender a inserção do ensino de Computação nas escolas, uma vez que os conceitos intrínsecos nessa área da ciência podem aprimorar a capacidade de solucionar problemas, além do suporte nas demais ciências do currículo. Nunes (2008) destaca que ao considerar Computação como um "saber" para a EB é necessário dar importância ao ensino de "noções de modelos computacionais, algoritmos, complexidade, autômatos, entre outros conteúdos", habilidades nomeadas por Wing (2006) de Pensamento Computacional (PC).

França e Tedesco (2015) elencam que "Ensinar habilidades computacionais na educação básica no Brasil pode, portanto, configurar-se um desafio e apresentar-se como um cenário repleto de oportunidades aos educadores, pesquisadores $e$ comunidade escolar". Não obstante, a preocupação de pesquisadores e educadores do mundo inteiro tem promovido a disseminação do PC no âmbito escolar, inclusive no Brasil. Essa ação toma proporção pois, ao considerar os fundamentos da computação como um aglomerado de "conceitos, habilidade e prática" que podem ser aplicados tanto em situações do dia-a-dia como nas diversas ciências, é possível realizar comparação do PC com as competências de ler, escrever e calcular (Wing, 2006). 
Gomes et al. (2013) exibiram uma solução com auxílio do Visual App Inventor para o ensino de lógica de programação no EM, objetivando a criação de aplicativos para a plataforma Android. Machado et al. (2010) apresentaram o projeto que focalizou em escolas do Ensino Fundamental e Médio a fim de estimular e evidenciar talentos para a área de Computação, enquanto isso, Garcia et al. (2008) expõem o projeto que insere alunos do EM no contexto do raciocínio lógico através de atividades baseadas em problemas, trabalhando com algoritmos e estruturas de dados. Aguiar et al. (2015) propôs o uso da robótica através do Projeto "Lego nas Escolas" para desenvolver o raciocínio lógico de alunos do Ensino Fundamental.

As possibilidades expostas demonstram que não há uma diretriz a ser seguida para o trabalho com PC no âmbito da EB, cada esforço possui suas características e metodologia singular, dificultando dessa maneira determinar seus impactos. França e Tedesco (2015) destacam que o principal elemento a ser considerado para efetivar a inserção do PC no ensino é o currículo escolar, destacando dois cenários para aplicação dos conceitos: um relacionado a criação de uma disciplina obrigatória no currículo, que aborde os fundamentos da Computação e outro difundido de maneira interdisciplinar nas disciplinas já inseridas.

Diferentemente da proposta de Aguiar et al. (2015), o trabalho aqui em destaque foi integrado ao currículo da $1^{\text {a }}$ Série de maneira obrigatória, conforme proposto por França e Tedesco (2015) e diferenciando das proposições de Machado et al. (2010) que focaram na busca por talentos. Outrossim, as ações realizadas objetivaram o desenvolvimento de talentos, realizando durante um ano letivo o desenvolvimento de atividades contextualizadas aplicadas através da robótica, o que favoreceu o aprendizado gradativo e contínuo de fundamentos do Pensamento Computacional, aplicando e afirmando as discussões de Rodrigues et al. (2015) e Wing (2006), além de aprimorar as proposições de Garcia et al. (2008).

\subsection{EBEP: O Ensino Básico do SESI}

Em 2014, o SESI-PB implantou nas cidades de Patos, Campina Grande, Bayeux e João Pessoa, o Programa Educação Básica articulada com Educação Profissional, juntamente com o Serviço Nacional de Aprendizagem Industrial (SENAI), composto por um currículo que contempla na $1^{\text {a }}$ Série, as disciplinas comuns a qualquer escola do Ensino Médio em um turno, e, no contraturno, participam de oficinas através das disciplinas transversais (parte integrante do currículo): Atualidades, Ciências Aplicadas, Projetos de Aprendizagem e Oficinas Tecnológicas. $\mathrm{Na} 2^{\mathrm{a}}$ Série, os alunos frequentam aulas regulares da educação básica em um período, e, no outro, o Curso Técnico Profissionalizante no SENAI.

Uma das disciplinas transversais mais importantes é a Oficina Tecnológica de Robótica. Em 2014, os alunos da 1 ${ }^{\text {a }}$ Série do SESI-PB de Bayeux, Campina Grande, João Pessoa e Patos iniciaram o contato através da Metodologia LEGO para o ensino da física. Não obstante, no decorrer das atividades, foi identificado que os alunos apresentavam dificuldade em desenvolver a etapa de programação dos robôs, impossibilitando muitas vezes a conclusão das atividades. A vulnerabilidade sinalizada pode ser originada de vários fatores, um deles está relacionado à ausência do contexto do PC em meio às formações dos professores. Considerando que o ENEM é pautado no 
V Congresso Brasileiro de Informática na Educação (CBIE 2016)

Anais do XXVII Simpósio Brasileiro de Informática na Educação (SBIE 2016)

raciocínio lógico, desenvolver uma proposta que contemple os conceitos do PC pode contribuir para o bom desempenho dos alunos nas 4 áreas do conhecimento.

\subsection{Modelo LEGO® de Educação Tecnológica}

O Modelo de Educação Tecnológica da LEGO® é baseado nos 4 pilares da educação: aprender a conhecer; aprender a fazer; aprender a viver juntos; $e$ aprender a ser (Delors, 2012). O modelo também considera uma competência maior: o aprender a agir. Além disso, foi idealizado para ser um instrumento de ajuda do docente em sala de aula e objetiva a construção de significado entre o que se ensina nas ciências, especialmente na física, e a realidade do aluno.

A metodologia é dividida em 4 fases (Contextualizar, Construir, Analisar e Continuar) e o modelo é ofertado sobre o aporte de materiais didáticos constituídos por Fascículos do Aluno, Manual do Educador e de Montagem. Os kits contêm atuadores, sensores, servos motores, conectores, engrenagens, vigas e softwares que permitem programar os robôs, a partir de blocos, favorecendo a imersão do aluno na programação dos mesmos. Essa combinação de objetos permite realizar atividades com temas contemporâneos relacionados à física, possibilitando o trabalho interdisciplinar.

\section{Metodologia}

Esse trabalho seguiu o método de pesquisa ação, caracterizando-se por envolver a identificação de um problema real, a colaboração de professores, a proposição de soluções, a proposição e implementação de intervenções e finalmente a avaliação dos resultados alcançados após a intervenção (Cohen et al., 2011). O desenvolvimento desse trabalho foi dividido em duas fases: 1) Desenvolvimento e aplicação de um curso de introdução ao Pensamento Computacional para professores e estudantes do ensino Médio da rede SESI-PB; 2) Análise do efeito do curso no desempenho de estudantes após a modificação da disciplina.

\subsection{Problemática e Intervenção}

O trabalho com a robótica iniciou em 2012 com a formação dos professores da área de exatas do SESI-PB, através de treinamentos divididos em 3 fases por ano. Mesmo havendo a necessidade de desenvolver programas para comandar os robôs, os treinamentos foram pautados em conceitos da física aliados à programação considerando exclusivamente as situações propostas, ou seja, o treinamento baseado na metodologia LEGO não contemplou conceitos de PC.

Em 2014, os alunos da $1^{a}$ Série do SESI-PB cursaram a Oficina de Robótica através da Metodologia LEGO para o ensino da física, sendo identificado que os alunos apresentavam dificuldade em desenvolver a etapa de programação dos robôs, impossibilitando muitas vezes a conclusão das atividades. Em junho de 2014 foi iniciado um treinamento com os professores para ensino da robótica utilizando o Pensamento Computacional, trabalhando conceitos de algoritmo, raciocínio lógico, linguagem de programação, todos aplicados à robótica através dos kits da LEGO. O trabalho foi desenvolvido por um Licenciado em Computação durante 6 meses, com 2 encontros presenciais por mês e atividades semanais a distância.

Os resultados evidenciados no desenvolvimento dos professores no que se refere ao uso aplicado da robótica foram satisfatórios. Eles se tornaram autônomos, passaram a 
V Congresso Brasileiro de Informática na Educação (CBIE 2016)

Anais do XXVII Simpósio Brasileiro de Informática na Educação (SBIE 2016)

desenvolver e programar seus próprios robôs, além disso, demonstraram ter absorvido a essência do PC, fato que possibilitou vislumbrar a necessidade de inserir o PC na Oficina Tecnológica de Robótica aplicada nas turmas da $1^{\mathrm{a}}$ Série. Em 2015 essa proposta foi incorporada na Oficina de Robótica, contemplando conceitos de arquitetura de computadores, robótica com enfoque nos equipamentos da LEGO, lógica de programação, algoritmos, linguagem de programação, tipos de dados, além de atividades com base em problemas de torneios e olimpíadas.

\subsection{Questões e Hipóteses de Pesquisa}

Para este trabalho buscou-se responder duas questões de pesquisa, a priori a questão RQ1 está relacionada análise do efeito da introdução do PC na oficina de robótica sob o desempenho de estudantes antes e após a modificação na disciplina. De forma análoga, RQ2 busca observar os efeitos da modificação da oficina sob a ótica das disciplinas básicas ministradas no Ensino Médio.

- RQ1: A introdução do Pensamento Computacional favorece o processo de ensino-aprendizagem na oficina de robótica?

- H1.0: Não há indícios de que a introdução de Pensamento Computacional na oficina de robótica melhora o desempenho de estudantes.

- H1.1: A introdução de Pensamento Computacional melhora o desempenho de estudantes na oficina de robótica.

RQ2: $O$ ensino de robótica com ênfase em PC pode aprimorar o desempenho de estudantes nas disciplinas básicas do Ensino Médio?

- H2.0: Não há indícios de que a introdução de PC na oficina de robótica influencia o desempenho de estudantes disciplinas do Ensino Médio.

- H2.1: Após a introdução de PC na oficina de robótica o desempenho de estudantes nas disciplinas básicas melhora significativamente em relação ao desempenho de alunos que participaram da antiga oficina.

\subsection{Amostra}

A amostra analisada consiste do desempenho de todos os estudantes aprovados na $1^{\text {a }}$ Série do Ensino Médio da rede SESI-PB (729 estudantes), nos anos de 2014 e 2015, nas cidades de Campina Grande, Bayeux e Patos. De acordo com os dados analisados, a amostra equivale a aproximadamente $84 \%$ dos alunos aprovados no estado da Paraíba na rede SESI. Além disso, os dados liberados pelo SESI são anônimos, respeitando critérios éticos e garantindo assim a privacidade dos alunos.

\subsection{Procedimentos para Análise dos Dados}

Para responder as questões de pesquisa caracterizamos os estudantes em dois grupos: experimental (277 alunos) e de controle (452 alunos); de acordo com o tipo de oficina de robótica (com ênfase em PC ou tradicional) que cada estudante cursou durante sua formação na $1^{\text {a }}$ Série do Ensino Médio. Para tratar o desbalanceamento dos grupos utilizamos o processo de reamostragem por reposição bootstrap proposto por Efron com 1000 iterações (EFRON, 1982).

Para análise estatística dos dados utilizamos a linguagem de programação R, um procedimento de análise gráfica e testes de hipótese foram utilizados para avaliar respectivamente a normalidade e homocedasticidade dos dados e assim nortear a escolha de testes estatísticos adequados para nossa análise. 
Diante do número de disciplinas no Ensino Médio envolvidas nessa análise foi realizado um procedimento de blocagem de disciplinas por áreas do conhecimento. Para isso, tomou-se como referência as 4 áreas de avaliação do ENEM.

O teste $t$ de student foi utilizado para avaliar se há diferença significativa entre o desempenho de ambos os grupos (experimental e de controle) na oficina de robótica, bem como nas demais disciplinas básicas do Ensino Médio. Para calcular e analisar o efeito da modificação da oficina sob o desempenho dos estudantes foi utilizado o índice de tamanho de efeito $d$ de Cohen (Cohen, 1992).

Para realização dos testes utilizamos um nível de confiança de $95 \%$ e significância estatística $\alpha=0.05$. Consideramos nessa análise os pressupostos estatísticos específicos para o teste $t$ (normalidade e homogeneidade das variâncias) e por meio de uma análise complementar garantimos a aleatoriedade dos dados tendo em vista a manutenção da validade dos resultados. Os leitores interessados podem consultar a análise dos pressupostos estatísticos neste link https://goo.gl/LpYBJV.

\section{Resultados e Discussão}

\subsection{RQ1: A Introdução do Pensamento Computacional Favorece o Processo de Ensino-Aprendizagem na Oficina de Robótica?}

Para responder a primeira questão de pesquisa (RQ1) a priori analisamos a média e desvio padrão dos grupos experimental e de controle na oficina de robótica. Como se observa na Tabela 3, a média do grupo experimental é 9,8\% maior em relação a média do grupo de controle. Além disso, o desvio padrão do desempenho dos alunos do grupo experimental é relativamente menor em relação a do grupo de controle, ou seja, há menor variação nas médias dos estudantes do grupo experimental em relação ao grupo de controle. Essa avaliação indica que há uma diferença real no desempenho dos grupos e um efeito positivo atribuído ao PC.

Para confirmar essa hipótese realizamos um teste de hipótese $t$ de student unicaudal com fator de correção de Welch (uma vez que essa área não apresentou homogeneidade nas variâncias) e nível de significância $\alpha=0.05$ considerando como hipótese alternativa que o desempenho dos estudantes do grupo experimental é superior ao de controle na oficina de robótica.

Com base no resultado obtido no teste (ver Tabela 3) podemos rejeitar a hipótese nula H1.0: Não há indícios de que a introdução de PC na oficina de robótica melhora o desempenho de estudantes com um nível de confiança de $95 \%$ uma vez que $p$-value < $\alpha$. Assim, assumimos a hipótese alternativa H1.1: A introdução de Pensamento Computacional melhora o desempenho de estudantes na oficina de robótica.

De forma complementar ao teste $t$ de student, o efeito da modificação da oficina sob o desempenho dos estudantes nos grupos experimental e de controle foi analisado.

Tabela 3 - Desempenho dos grupos, teste de hipótese e efeito para oficina de robótica

\begin{tabular}{|c|c|c|c|c|c|c|c|}
\hline \multirow{2}{*}{ Disciplina } & \multicolumn{2}{|c|}{ Experimental (E) } & \multicolumn{2}{|c|}{ Controle (C) } & \multirow{2}{*}{$\begin{array}{l}\text { Diferença } \\
\text { média \% }\end{array}$} & \multirow{2}{*}{ t-student } & \multirow{2}{*}{ Efeito (d) } \\
\hline & Média & DP & Média & DP & & & \\
\hline Robótica & 78 & 4 & 71 & 7 & $9,8 \%$ & $<0.001$ & 1.25 \\
\hline
\end{tabular}


Calculando o índice de tamanho de efeito $d$ de Cohen obtemos $d=1.25$, como $d$ é exatamente equivalente ao Z-score tabelado de uma distribuição normal padrão (Coe, 2002) a partir da consulta na tabela normal padrão temos que $89 \%$ dos alunos do grupo experimental apresentam uma média superior em relação ao grupo controle, seguindo a definição de Cohen (1992) esse efeito é alto uma vez que $d>0.8$.

\subsection{RQ2: O ensino de robótica com ênfase em Pensamento Computacional pode aprimorar o desempenho de estudantes nas disciplinas básicas do Ensino Médio?}

Para responder a segunda questão de pesquisa (RQ2), primeiramente analisamos a média e desvio padrão dos grupos experimental e de controle de cada área do conhecimento avaliada no Ensino Médio. De acordo com a Tabela 4, a média do grupo experimental se mostra maior em todas as áreas analisadas em relação ao grupo de controle, já o desvio padrão parece constante para cada área avaliada em ambos os grupos.

Realizamos um teste de hipótese $t$ de student unicaudal utilizando nível de significância $\alpha=0.05$ considerando como hipótese alternativa que o desempenho dos estudantes do grupo experimental é superior ao de controle na oficina de robótica. $\mathrm{O}$ fator de correção de Welch foi aplicado apenas para as áreas de Linguagens e Ciências da Natureza, pois as variâncias nessas áreas não são homogêneas.

Tabela 4 - Desempenho dos grupos por área de conhecimento do Ensino Médio, teste de hipótese e efeito

\begin{tabular}{|c|c|c|c|c|c|c|c|}
\hline \multirow{2}{*}{$\begin{array}{c}\text { Área do } \\
\text { conhecimento }\end{array}$} & \multicolumn{2}{|c|}{ Experimental (E) } & \multicolumn{2}{|c|}{ Controle (C) } & \multirow{2}{*}{$\begin{array}{l}\text { Diferença } \\
\text { média \% }\end{array}$} & \multirow{2}{*}{$\begin{array}{c}t- \\
\text { student }\end{array}$} & \multirow{2}{*}{ Efeito (d) } \\
\hline & Média & DP & Média & DP & & & \\
\hline Matemática & 70 & 13 & 65 & 12 & $7,6 \%$ & $<0.001$ & 0.34 \\
\hline Linguagens & 75 & 8 & 72 & 6 & $4,1 \%$ & $<0.001$ & 0.43 \\
\hline Humanas & 76 & 7 & 68 & 7 & $11,7 \%$ & $<0.001$ & 1.19 \\
\hline Natureza & 73 & 9 & 68 & 8 & $7,3 \%$ & $<0.001$ & 0.54 \\
\hline
\end{tabular}

As saídas obtidas nos testes de hipótese $t$ de student são apresentadas na Tabela 4. Como se observa para todas as áreas do conhecimento avaliadas temos $p$-value $<\alpha$, assim com um nível de confiança de 95\% rejeitamos a hipótese nula H2.0: Não há indícios de que a introdução de Pensamento Computacional na oficina de Robótica influencia o desempenho de estudantes disciplinas do Ensino Médio. Logo, assumimos a hipótese alternativa H2.1: Após a introdução de PC na oficina de robótica o desempenho de estudantes nas disciplinas básicas melhora significativamente em relação ao desempenho de alunos que participaram da antiga oficina.

O efeito da modificação da oficina sob o desempenho dos estudantes nas áreas do conhecimento avaliadas no Ensino Médio foi positivo. Com base no índice de tamanho de efeito $d$ de Cohen calculado para cada área, como pode ser observado na Tabela 4, verificamos por meio da consulta na tabela normal que estudantes do grupo experimental apresentaram médias superiores em relação ao grupo de controle nas seguintes proporções e áreas: $63 \%$ Matemática (efeito pequeno $0.2<d<0.5$ ), 67\% Linguagens (efeito pequeno $0.2<d<0.5$ ), $88 \%$ Ciências Humanas (efeito alto $d>0.8$ ) e $70 \%$ Ciências da Natureza (efeito médio $0.5<d<0.8$ ); a classificação do efeito foi baseada na definição de Cohen (1992).

De modo geral, considerando as habilidades e competências exploradas pelo PC, resultados quantitativos positivos mediante a ênfase em PC estabelecida na oficina de 
V Congresso Brasileiro de Informática na Educação (CBIE 2016)

Anais do XXVII Simpósio Brasileiro de Informática na Educação (SBIE 2016)

robótica eram esperados. Os resultados dispostos nessa seção são compatíveis com a literatura teórica e qualitativa sobre o tema.

\subsection{Ameaças à Validade}

Validade de Constructo: o desenvolvimento do curso, bem como as intervenções foram realizadas e validadas por especialistas da área de educação e computação. Os dados analisados foram fornecidos pelo SESI-PB garantindo a confiabilidade.

Validade Interna: Nesse estudo analisamos amostras aleatórias representativas da população de estudantes da $1^{\text {a }}$ Série do Ensino Médio do SESI-PB, com isso buscamos diminuir o viés e garantir a validade interna dos resultados. Contudo, nesse trabalho não foi possível controlar fatores da vida acadêmica que podem influenciar a análise como, por exemplo, dependência administrativa da escola, qualidade do Ensino Fundamental e atividades extracurriculares realizadas.

Validade de Conclusão: Consideramos todos os pressupostos dos testes estatísticos utilizados nesse trabalho, bem como utilizamos amostras com características semelhantes para composição dos grupos avaliados na análise.

Validade Externa: A amostra analisada é representativa da população de estudantes da $1^{\text {a }}$ Série do Ensino Médio do SESI-PB, logo, ainda não podemos generalizar os resultados alcançados para outras populações de estudantes.

\section{Conclusão}

Provindo das adversidades evidenciadas no decorrer do ensino da disciplina Oficina de Robótica, na $1^{\text {a }}$ Série do EM do SESI-PB, sem ligação com o PC, foi realizado um trabalho em duas fases. A primeira constituiu o desenvolver e aplicar um curso introdutório ao Pensamento Computacional aplicado à robótica para professores e estudantes do Ensino Médio do SESI-PB. A segunda foi relacionada à análise do efeito do curso no desempenho de estudantes após a modificação da proposta curricular.

A análise evidenciou que a inserção de PC melhora o desempenho de estudantes na Oficina de Robótica. Além disso, o contato com Pensamento Computacional como parte integrada de disciplinas do EM melhora o desempenho de estudantes nas disciplinas básicas de maneira significativa. Os resultados destacam que a inserção de PC no EB é capaz de auxiliar o desenvolvimento do aluno de modo geral, não obstante, não podemos generalizar os resultados alcançados para outras populações de estudantes, pois analisamos dados de estudantes da $1^{\text {a }}$ Série do EM do SESI-PB.

Esperamos que as questões apresentadas neste trabalho corroborem para os direcionamentos da inserção do Pensamento Computacional no Ensino Básico e, como pesquisas futuras, desejamos realizar nova análise dos dados a fim de avaliar os impactos da inserção do PC no EM do SESI-PB por disciplina. Com isso, pretendemos identificar possibilidades e/ou melhorias do uso de Pensamento Computacional para impactar de maneira mais significativa naquelas em que o impacto não foi expressivo. Além disso, pretendemos efetivar um novo estudo com alunos das próximas turmas da $1^{a}$ Série do SESI-PB, a fim de acompanhar o desenvolvimento das habilidades destacadas nos direcionamentos do SEB para o Ensino Básico, avaliando os alunos no início do ano letivo de 2017, antes de iniciar a Oficina Tecnológica de Robótica e ao término do ano, após a vivência do conteúdo programático com PC. 
V Congresso Brasileiro de Informática na Educação (CBIE 2016)

Anais do XXVII Simpósio Brasileiro de Informática na Educação (SBIE 2016)

\section{Referências}

Aguiar, Y. Q., Maciel, B. K., Matos, S. D. G., Soares, L. B., Oliveira, V. M. (2015). Introdução à Robótica e Estímulo à Lógica de Programação no Ensino Básico Utilizando o Kit Educativo LEGO ${ }^{\circledR}$ Mindstorms. In: Anais dos Workshops do CBIE 2015. SBC, p. $1418-1424$.

Cohen, J. (1992). A power primer. Psychological bulletin, 112(1): 155.

Cohen, L., Manion, L., and Morrison, K. (2011). Research Methods in Education. Education, Research methods. Routledge.

Coe, R. (2002). It's the effect size, stupid: What effect size is and why it is important. Disponível em: $<$ http://www.leeds.ac.uk/educol/documents/00002182.htm>. Acesso em: 30 de maio de 2016.

CSTA (2016). Computational thinking teacher resources. Disponível em: $<$ http://www.csta.acm.org/Curriculum/sub/CompThinking.html $>$. Acesso em: 06 de junho de 2016.

Efron, B. (1982). The jackknife, the bootstrap and other resampling plans. Vol. 38. Philadelphia: Society for industrial and applied mathematics.

França, R. S., Tedesco, P. C. A. R. (2015). Desafios e Oportunidades ao Ensino do Pensamento Computacional na Educação Básica no Brasil. In: Anais dos Workshops do CBIE 2015. SBC, p. 1464-1473.

Garcia, R. E., Correia, R. C. M., Shimabukuro, M. H. (2008). Ensino de Lógica de Programação e Estruturas de Dados para Alunos do Ensino Médio. In: Anais do WEI 2008. SBC.

Gomes, T. C. S.; Melo, J. C. B. (2013) O Pensamento Computacional no Ensino Médio: Uma Abordagem Blended Learning In: Anais do WEI 2013. SBC.

INEP (2016). Conteúdo das Provas (2016). Disponível em: $<$ http://inep.gov.br/web/enem/conteudo-das-provas $>$. Acesso em: 01 de maio de 2016.

Delors, J. (2012). Educação: um tesouro a descobrir - Relatório para a Unesco da Comissão Internacional sobre Educação para o século XXI. Cortez.

Oliveira, M. K. (2000). Vygotsky, aprendizado e desenvolvimento um processo sóciohistórico. $4^{\mathrm{a}}$ ed. São Paulo. Scipione, p. 111.

Lopes, A. C. (2005), Recontextualização e Hibridismo. Currículo sem fronteiras, v. 5, n. 2 , p. 50-64.

Machado, E. Z. A., Vasconcelos, I. R., Amorim, K. M., Andrade, A. M. S. (2010). Uma Experiência em Escolas de Ensino Médio e Fundamental para a Descoberta de Jovens Talentos em Computação. In: Anais do WEI 2010. SBC, p. 798-807.

Matos, E. S. (2013) Integração curricular por meio da prática de ensino interdisciplinar em IHC. In: Anais do WEIHC 2013. Manaus, AM. p. 25-30.

Nunes, D. J. (2008). Licenciatura em Computação. Jornal da Ciência, 30 de maio.

Paiva, L. F., Ferreira, A. C., Barreto, J., Lopes, R., Matos, E. (2015). Uma Experiência Piloto de Integração Curricular do Raciocínio Computacional na Educação Básica. In: Anais dos Workshops do CBIE 2015. SBC, p. 1300-1309.

Rodrigues, R. S., Andrade W. L., Guerrero, D. S., Sampaio, L. M. R. (2015). Análise dos efeitos do Pensamento Computacional nas habilidades de estudantes no ensino básico: um estudo sob a perspectiva da programação de computadores. In: Anais do XXVI Simpósio Brasileiro de Informática na Educação. 2015. p. 121.

Wing, J. M. (2006). Computational thinking. Commun. ACM, 49(3):33-35. 